\title{
THAI CONSENSUS ON VENOUS THROMBOEMBOLISM IN HIP AND KNEE SURGERY
}

\author{
Saradej Khuangsirikul*, Viroj Larbpaiboonpong**, Charlee Sumettavanich ***, Surapoj Meknavin****, \\ Aree Tanavalee*****, Thanainit Chotanaphuti* \\ * Department of Orthopaedics, Phramongkutklao College of Medicine, Bangkok, Thailand \\ ** Orthopedic Department, Police General Hospital, Bangkok, Thailand \\ *w* Department of Orthopaedics, Lerdsin General Hospital, Bangkok, Thailand \\ ***** Department of Orthopaedics, Faculty of Medicine Vajira Hospital, Navamindradhiraj University, Bangkok, \\ Thailand \\ $* * * * *$ Department of Orthopaedics, Faculty of Medicine Chulalongkorn University, Bangkok, Thailand
}

\section{Chairman: Saradej Khuangsirikul}

Delegates: Chaithavat Ngarmukos, Wallob Samranvethya, Thanainit Chotanaphuti, Aree Tanavalee, Surapoj Meknavin, Viroj Larbpaiboonpong, Charlee Sumettavanich, Srihatach Ngarmukos, Satit Thiengwittayaporn, Nattapol Tammachote, Siwadol Wongsak, Saradej Khuangsirikul, Apisit Patamarat, Samart Muangsiri, Tulpong Ampool, Worapoj Honglerspipop, Chaturong Pornrattanamaneewong, Aasis Unnanuntana, Sukree Khumrak, Ukrit Chaweewannakorn, Wiboon Wanitcharoenporn, Burin Sutthapakti, Kreangsak Lekkreusuwan

\begin{abstract}
Increasing numbers of hip and knee arthroplasty may associate with higher risk of complications such as venous thromboembolism. Most of Thai orthopedic surgeons ignore thromboprophylaxis because of less symptomatic VTE in Asian population. The "Thai consensus on venous thromboembolism in hip and knee surgery" consist of twenty-four common questions about VTE including their supported literatures. The gold standard of diagnosis is contrast venography although the duplex ultrasound has more role in postoperative patients. Thai patients undergoing hip and knee surgery seem to have lower prevalence of VTE than Caucasian. However, mechanical or chemoprophylaxis should be considered especially in the high risk group and hip fracture in elderly.
\end{abstract}

Keywords : Venous thromboembolism, Deep vein thrombosis, Hip and knee arthroplasty, Thromboprophylaxis, Chemoprophylaxis

J Southeast Asian Med Res 2017;1:29-43.

http://www.jseamed.org

\section{Correspondence to:}

Chotanaphuti T, Department of Orthopaedics, Phramongkutklao College of Medicine, Bangkok, Thailand E-mail : tanainit@hotmail.com 


\section{Introduction}

Higher life expectancy in Thai population is associated with increasing of the degenerative disorders. The numbers of osteoarthritis of hip and knee that non-response to conservative treatment, such as medication and physical therapy, are also increasing. Hip and knee arthroplasty in elderly are the major operations which not only improve the quality of life, but also able to create some complications. One of the common complications following hip and knee surgery is venous thromboembolism (VTE) which has several degrees of severity (asymptomatic-life-threa tening).

According to awareness of VTE sequelae, the "Thai consensus conference on venous thromboembolism in hip and knee surgery 2016" was held on $15^{\text {th }}-17^{\text {th }}$ September, 2016 at Lampang, Thailand by the Thai Hip and Knee Association. The participants are Thai orthopedic surgeons who work in different institutions including government hospitals, universities, military services and private hospitals. Modified Delphi method was applied to generate four categories of questions and answers; 1) diagnosis of deep vein thrombosis (DVT)/VTE, 2) risk of DVT/VTE, 3) prevention, and 4) chemoprophylaxis. Finally, twenty-four questions and answers were voted for agreement.

The level-1 evidence-based literature were reviewed by the experts to respond all questions, establishing a consensus. Although the data included in this consensus are suitable for most of Thai patients who undergoing hip and knee surgery. In some situations, the surgeons might have to make different decision for appropriate management.

Question 1: Which investigation is the gold standard for diagnosing DVT after TJA?

Consensus: Contrast venography.

Delegate vote: Agree: $100 \%$, Disagree: $0 \%$, Abstain: $0 \%$ (Unanimous Consensus)

\section{Justification:}

Deep venous thrombosis (DVT) continues to be a source of major morbidity and mortality for surgical patients. The incidence of postoperative DVT is as high as $28 \%$ in some series. Indeed, the clinical complications (from postthrombotic syndrome to fatal pulmonary embolism) as well the risk of anticoagulant treatment require a precise diagnosis. Since clinical diagnosis of DVT is barely sensitive and specific.
Thus, the clinical suspicion of DVT needs to be confirmed by objective testing. Venography has been for a long time the reliable technique to confirm or rule-out the clinical suspicion of DVT. Furthermore, venography served as "gold standard" for the validation of diagnostic methods for DVT. ${ }^{(1,2)}$ However, venography is invasive, expensive, not always available, and occasionally inadequate or risky, noninvasive diagnostic strategies for diagnosing DVT have been developed. Non-invasive diagnostic methods (venous ultrasonography) are sufficiently sensitive for venous occlusions. The optimal strategy at individual institutions is dependent on local expertise and cost. ${ }^{(1,3)}$

Question 2: Should isolated leg swelling be investigated for DVT after TJA?

Consensus: Isolated swelling in the operated limb without other signs of deep vein thrombosis does not require investigation.

Delegate vote: Agree: $95.8 \%$, Disagree: $4.2 \%$, Abstain: $0 \%$ (Strong Consensus)

\section{Justification:}

Swelling of operated leg is very common after THA and TKA. The postoperative swelling can be caused by direct injury from surgery as well as penetration of intra-articular blood collection into surrounding soft tissue. Although leg swelling is one of clinical signs of deep vein thrombosis, isolated leg swelling after THA and TKA should not be used as an indication for investigation unless other signs of DVT are also presented. Clinical signs of DVT include: ${ }^{(4)}$

- Swelling

- Pain or tenderness

- Distended veins

- Red or discolored skin

- Firmness or thickening of the vein

Very few clinical studies report correlation between incidence and clinical sings of DVT after THA and TKA. A retrospective study about risk factors for limb swelling after TKA by Gao et al. reveals no correlation between leg swelling (increased circumference) and incidence of DVT. ${ }^{(5)}$ Since there are no strong supporting evidences, decision has to be made based on expert opinion by considering unclear benefit of venographic study in this situation and financial cost of investigation. 
Question 3: Does D-dimer have a role for diagnosing VTE after TJA?

Consensus: D-dimer does not have a role for diagnosing VTE after TJA.

Delegate vote: Agree: $95.8 \%$, Disagree: $4.2 \%$, Abstain: $0 \%$ (Strong Consensus)

\section{Justification:}

D-dimer is the degradation product of crosslinked (by factor XIII) fibrin. ${ }^{(6-13)}$ It is a marker of endogenous fibrinolysis and should therefore be detectable in patients with deep vein thrombosis (DVT). ${ }^{(14-18)}$ Several studies have shown the d-dimer assay to have a high negative predictive value and d-dimer to be a sensitive but nonspecific marker of deep-vein thrombosis. ${ }^{(6-8)}$ D-dimer is a very useful diagno- stic tools for non-surgical patients, however post operation especially in hip and knee surgery, d-dimer result will be all in positive value which ultrasonography confirmation shown to be clear of DVT. ${ }^{(9)}$ There are at least three evidence studies that suggest d-dimer is not useful screening test for VTE or DVT after arthroplasty. Two studies used ultra- sound as reference, while one used venography.

Question 4: Should duplex ultrasound be used as a screening test for diagnosing VTE in clinically suspected patient?

Consensus: Yes.

Delegate vote: Agree: 91.6\%, Disagree: 4.2\%, Abstain: $4.2 \%$ (Strong Consensus)

\section{Justification:}

The gold standard for VTE diagnosis is venography, but it is not proper for VTE screening in all patients because of cost and the invasiveness. The investigation that is less invasive than venography and widely used to diagnose VTE is duplex ultrasound. From the study of duplex ultrasound in the diagnosis of VTE in patients suspected to have VTE had sensitivity and specificity of $95 \%$ and $99 \%$, respectively $^{(19,20)}$, but the value will be reduced to $31.1 \%$ and $93 \%$, respectively, in patients who are not suspected VTE. ${ }^{(21,22)}$ Hence, the use of duplex ultrasound for DVT screening should be conducted only in the patients that suspected of DVT. The advantages of duplex ultrasound are inexpensive and less invasive to the patient with high sensitivity and specificity.
However, the duplex ultrasound requires an experienced radiologist. The disadvantages of using duplex ultrasound in VTE screening are operator dependent and have low sensitivity in patients who are not suspected DVT.

Question 5: Is it necessary to perform a routine screening test for DVT in patients undergoing TJA?

Consensus: No, it is not necessary to perform a routine screening test for DVT in patients undergoing TJA.

Delegate vote: Agree: $100 \%$, Disagree: $0 \%$, Abstain: $0 \%$ (Unanimous Consensus)

\section{Justification:}

We do not recommend the routine use of duplex ultrasound or contrast venography for screening of DVT in the patients undergoing hip or knee arthroplasty. The AAOS guideline ${ }^{(23)}$ and two randomized controlled studies $^{(24,25)}$ found no statistically significant difference in symptomatic PE incidence between screening and non- screening group. The available evidences also suggest that d-dimer is not a useful screening test for DVT after arthroplasty, these studies use ultrasound and venography as the standard reference. ${ }^{(10-12)}$

Question 6: Which factors increase the risk of VTE in TJA patient?

Consensus: Cancer, previous VTE, delayed ambulation ( $>4$ days), Caucasian, a family history of VTE, hypercoagulable state, hormone replacement therapy, and multiple comorbidities

Delegate vote: Agree: $100 \%$, Disagree: $0 \%$, Abstain: $0 \%$ (Unanimous Consensus)

\section{Justification:}

Patients undergoing elective hip or knee arthroplasty are at high risk for venous thromboembolic disease (VTE) manifesting as deep vein thrombosis (DVT) or pulmonary embolism (PE). Previous history of VTE is the only risk factor that has sufficient evidence indicating that some of these patients may be at even higher risk. ${ }^{(26)}$ Although a previous study in elective hip and knee arthroplasty patients has not clearly demonstrated whether factors other than a previous history of VTE increase the risk of VTE, we still believe that these potential risk factors including cancer, delayed ambulation ( $>4$ days), Caucasian race, family history of VTE, hypercoagulable state, hormone 
replacement therapy and multiple comorbidities may increase the risk of VTE. The practitioner should consider making a prophylaxis of VTE in patients with these risk factors.

Question 7: Does obesity increase the risk of VTE in TJA? Consensus: It is controversial whether obesity increases the risk of VTE in TJA.

Delegate vote: Agree: $87.4 \%$, Disagree: $4.2 \%$, Abstain: $8.4 \%$ (Strong Consensus)

\section{Justification:}

According to World Health Organization (WHO) criteria established in $1997^{(27)}$, obesity among adults is further classified into three categories: class I obesity is a BMI of $30-34.9 \mathrm{~kg} / \mathrm{m}^{2}$; class II is a BMI of $35-39.9 \mathrm{~kg} / \mathrm{m}^{2}$; and class III, or morbid obesity, is a BMI $\geq 40 \mathrm{~kg} / \mathrm{m}^{2}$. Increasing BMI above normal value has been reported to be associated with a rising risk of VTE. ${ }^{(28-30)}$ Some studies have indicated that mortality among patients with $\mathrm{PE}$ appeared to be paradoxically lower among patients who were obese than those who were not. ${ }^{(31,32)}$

However, it remains obscure whether this phenomenon the so-called "obesity paradox" ${ }^{(33)}$ is attributable to a real protective role of increased body fat because the lower mortality was seen mostly among old people who were obese, but not among young adults and children who were obese. $^{(31,32)}$ By analyzing the relationship of body fat (\%) with VTE, a significant association was found in women, but not for men. ${ }^{(34)}$ In summarized, there is the relationship of obesity with some common weak-to moderate risk factors for VTE, including genetic factors, use of sex steroid hormones, inflammation, and insulin resistance. ${ }^{(35,36)}$

Question 8: Does Thai population have lower prevalence of DVT than Caucasian after TJA?

Consensus: Thai population seems to have lower prevalence of DVT than Caucasian after TJA.

Delegate vote: Agree: $95.8 \%$, Disagree: $4.2 \%$, Abstain: $0 \%$ (Strong Consensus)

\section{Justification:}

Prevalence of DVT after TKA without any prophylaxis for DVT was reported range from 40 to $88 \%$ among the Caucasian population. ${ }^{(37)}$ While prevalence of DVT after THA without prophylaxis in Caucasian was reported range from 8 to $70 \%{ }^{(38)}$ Among of this, proximal DVT was reported around $20 \%$ in the western world. For Asian countries including Thailand, prevalence of DVT was reported differently from the western world. From meta-analysis, prevalence of DVT following hip surgery and knee arthroplasty were $25.8 \%$ and $42.5 \%$, respectively. Rate of proximal DVT of $9.6 \%$ (hip surgery) and $8.7 \%$ (knee arthroplasty) were reported in this meta-analysis. ${ }^{(39)}$

Question 9: Should chemoprophylaxis be administrated in Thai patient undergoing total joint arthroplasty?

Consensus: Yes, chemoprophylaxis should be administrated in high-risk Thai patient.

Delegate vote: Agree: 95.8\%, Disagree: $0 \%$, Abstain: $4.2 \%$ (Strong Consensus)

\section{Justification:}

As we known, patients who undergoing total joint arthroplasty are at high risk for venous thromboembolism. ${ }^{(26)}$ In Thai patients, the incidences of overall and proximal deep vein thrombosis that detected by using venography after total knee arthroplasty are $61 \%$ and $12 \%$, respectively. ${ }^{(40)}$ To prevent these complications, we agree to use mechanical prophylaxis in all patients. Because of the two reasons, first, we agree that Thai population has lower prevalence of symptomatic venous thromboembolism than Caucasians ${ }^{(39)}$, and second, bleeding complications of chemoprophylaxis, we therefore suggest using the chemoprophylaxis in high-risk patients. The risk factors are justified in above mention.

Question 10: Does tranexamic acid increase the risk of VTE in TJA patient?

Consensus: Tranexamic acid does not increase the risk of VTE in TJA patient.

Delegate vote: Agree: $100 \%$, Disagree: $0 \%$, Abstain: $0 \%$ (Unanimous Consensus)

\section{Justification:}

Tranexamic acid (TXA) is an antifibrinolytic drug used for reducing blood loss in many surgical specialties. Meta-analysis and RCTs conclude that TXA can reduce blood loss, not increase incidence of DVT complications, and should be considered for routine use in primary total knee arthroplasty. ${ }^{(41-43)}$ The route of administration and dosage do not appear to affect complication rates either. 
Question 11: Does hip fracture patient carry the same risk of VTE as elective TJA patient?

Consensus: Hip fracture patient does not carry the same risk of VTE as elective TJA patient.

Delegate vote: Agree: $87.4 \%$, Disagree: $8.4 \%$, Abstain: $4.2 \%$ (Strong Consensus)

\section{Justification:}

Venous thromboembolism (VTE) remains a substantial cause of morbidity and mortality following hip fracture. Asymptomatic deep vein thrombosis (DVT) has been reported in up to $50 \%$ of all patients who sustain a hip fracture, with an incidence of fatal pulmonary embolus (PE) of up to $10 \%{ }^{(4-46)}$

From the study of Hefley FG Jr et al., they found Thirteen (10 percent) of 133 patients who had venography on admission to the hospital for a fracture about the hip had radiographic evidence of deep-vein thrombosis. ${ }^{(47)}$ Only seven ( 6 percent) of the 122 patients who were seen at the hospital within two days after the fracture had evidence of thrombosis. However, six of the eleven patients who had a delay of more than two days between the fracture and admission to the hospital had evidence of thrombosis.

The study from Korea examined the incidence and trends of clinically relevant VTE including DVT and pulmonary embolism (PE) after hip and knee replacement arthroplasty (HKRA) in Korea. The overall incidence of VTE, DVT, and PE during 90 days were $3.9 \%(\mathrm{n}=853), 2.7 \%(\mathrm{n}=597)$ and $1.5 \%(\mathrm{n}=327)$ after HRA, while the incidence was $3.8 \%$ $(\mathrm{n}=1,990), 3.2 \%(\mathrm{n}=1,699)$, and $0.7 \%(\mathrm{n}=355)$ after $\mathrm{KRA}^{(48)}$

Question 12: Does regional anesthesia lower the risk of venous thromboembolism comparing to general anesthesia in total joint arthroplasty?

Consensus: Yes, regional anesthesia has a lower risk of venous thromboembolism than general anesthesia.

Delegate vote: Agree: $79.2 \%$, Disagree: $0 \%$, Abstain: $20.8 \%$ (Strong Consensus)

\section{Justification:}

Regional anesthesia, including epidural and spinal anesthesia, has several potential advantages over general anesthesia in total joint arthroplasty. From the meta-analysis of 21 independent randomized trials, regional anesthesia can be beneficial in reducing the duration of surgery, the requirement of transfusion, postoperative nausea and vomiting. ${ }^{(49)}$ In terms of venous thromboembolism, regional anesthesia has a lower incidence of deep vein thrombosis and pulmonary embolism when compared to general anesthesia. However, the effects of regional anesthesia on long-term functional outcome after total joint arthroplasty are still unknown due to the lack of high quality evidence. ${ }^{(50)}$

Question 13: Does tourniquet time (or operative time) affect the risk of VTE in TJA patient?

Consensus: Prolonged tourniquet time (or operative time) increases the risk of VTE in TJA patient.

Delegate vote: Agree: $87.4 \%$, Disagree: $8.4 \%$, Abstain: $4.2 \%$ (Strong Consensus)

\section{Justification:}

TJA is one of the risk factors of DVT that have post-operative incidence of $20.3 \%-84 \% .{ }^{(51-53)}$ We found that operative time was considered to be significantly associated with the incidence of proximal DVT. ${ }^{(54)}$ Data show that a total operative time which more than 2 hours is correlated with DVT. Incidence of DVT increase when tourniquet time exceeding 60 minutes, age older than 50 years and previous history of DVT.

Question14: Does mechanical prophylaxis have a role in all TJA patients?

Consensus: Yes, mechanical prophylaxis should be performed in all TJA patients.

Delegate vote: Agree: $100 \%$, Disagree: $0 \%$, Abstain: $0 \%$ (Unanimous Consensus)

\section{Justification:}

Mechanical prophylaxis for venous thromboembolism (VTE) act on venous stasis. Mechanical prophylaxis can be static (the graduated compression stockings, elastic stockings or anti-embolism stockings), dynamic (intermittent pneumatic compression and venous foot pump) or both. The main advantage of these methods is lack of bleeding risk. Mechanical prophylaxis is recommended for every patients especially in patients who have high bleeding risk. $^{(55,56)}$ Mechanical and pharmacological methods of VTE prophylaxis are both effective and when used in combination have synergistic effects. ${ }^{(57)}$ 
Question 15: Does intermittent pneumatic compressive device (IPCD) have a role for preventing VTE in TJA patient?

Consensus: IPCD have a role for preventing VTE in TJA patient.

Delegate vote: Agree: 100\%, Disagree: 0\%, Abstain: 0\% (Unanimous Consensus)

\section{Justification:}

Chemoprophylaxis has been recommended to reduce the prevalence of postoperative DVT on the assumption that it will reduce the prevalence of PE, mortality, and thrombophlebitic syndrome. However, chemoprophylaxis carries a bleeding risk, with associated complications of blood loss, blood transfusion, transfusion-related transmission of disease, wound-healing problems, hematoma, slowed rehabilitation, wound drainage, and infection. ${ }^{(58-61)}$

IPCD is a mechanical device which is not only decrease the venous stasis and increase the blood flow in the lower extremities through external compression, but also can be a good option for avoid bleeding complication. In Asian people, Kim et al, found that patient who received treatment with a mechanical compression device only for prevention of DVT after primary TKA, the prevalence of DVT was only $6.6 \%$ compare to Lee et al, who report the incidence of DVT without thromboprophylaxis was $40.4 \% .{ }^{(62,63)}$ In a meta-analysis of Pour et al, IPCDs are effective in prevention of venous thromboembolic disease after total hip and knee arthroplasty compared to chemoprophylaxis. ${ }^{(64)}$

Also, the meta-analysis of Westrich et al, the incidence of DVT after TKA in the patients receiving IPCD was $17 \%$ that was significant lower than the patients receiving warfarin $(45 \%)$ or aspirin $(53 \%){ }^{(53)}$ Both AAOS and ACCP guideline agree that mechanical prophylaxis is appropriated. ${ }^{(23)}$ However, there has been a paucity of randomized clinical trial on its efficacy without chemoprophylaxis, therefore ACCP guideline suggest combining chemoprophylaxis with mechanical compression during hospitalization. ${ }^{(65)}$

Question 16: Which types of chemoprophylaxis are effective for preventing VTE in TJA patient?

Consensus: Aspirin, direct oral anticoagulants (DOAC), vitamin $\mathrm{K}$ antagonist and low-molecular-weight heparin (LMWH)
Delegate vote: Agree: $100 \%$, Disagree: $0 \%$, Abstain: $0 \%$ (Unanimous Consensus)

\section{Justification:}

According to ACCP guideline, aspirin has been recommended for reducing VTE event in hip and knee replacement. ${ }^{(65)}$ The recent systematic review, including 11 relevant studies with variation of dose regiments, concluded that aspirin can reduce VTE with low risk of bleeding complication. ${ }^{(6)}$ Another large trial (PEP trial) including 13,356 patients underwent surgery for hip fracture and elective hip arthroplasty with use of $160 \mathrm{mg}$ aspirin compare with placebo, they concluded that aspirin can reduce PE of $43 \%$ and symptomatic DVT of $29 \%$ without increasing death due to bleeding ${ }^{(67)}$ However, aspirin group increase postoperative transfused bleeding episodes significantly. In compare with other agents, Drescher FS et al included 8 trials compare with anticoagulants and found that aspirin trend to associate with higher risk of VTE and lower risk of bleeding but there was no statistical significant. ${ }^{(68)}$ For economic aspect, Schousboe JT et al showed aspirin is a cost-effective choice for VTE prophylaxis following total hip arthroplasty in patient without history of VTE. ${ }^{(69)}$

Direct factor Xa inhibitors work by binding to the active site of factor $\mathrm{Xa}$, thus, blocking the interaction with its substrate. Examples of oral direct factor Xa inhibitors are rivaroxaban, apixaban, edoxaban, and betrixaban. Rivaroxaban and apixaban are recommended by the ACCP in the same manner as the fondaparinux. ${ }^{(65)}$ Rivaroxaban is a FDA approved oral direct factor Xa inhibitor that requires no monitoring. Lassen et al. reported that $10 \mathrm{mg}$ of rivaroxaban taken once daily was more effective than $40 \mathrm{mg}$ of enoxaparin administered once daily in reducing overall VTE for TKA patients $(\mathrm{RR}=9.2 \% ; p<0.001)$. ${ }^{(70)}$ There was no difference in major bleeding between rivaroxaban and enoxaparin groups $(0.6 \%$ vs $0.5 \% ; p>0.05)$.).

In a study comparing $10 \mathrm{mg}$ of rivaroxaban taken 6 hours postoperatively against $40 \mathrm{mg}$ enoxaparin administered on the preoperative evening as prophylaxis for THA, rivaroxaban was significantly more effective than enoxaparin in preventing total VTE (1.1\% vs 3.7\%, RR of $2.6 \% ; p<0.001)$ but not in symptomatic events $(96.8 \%$ vs $97.0 \% ; p<0.05$ ). 
No difference in occurrence of major bleeding $(p=0.18)$. $^{(71)}$

Apixaban is a direct oral factor Xa inhibitor that has not been approved by FDA in United States. Lassen et al. conducted a series of studies comparing apixaban against different doses of enoxaparin as prophylaxis for total joint arthroplasty patients. For TKA patients, when $2.5 \mathrm{mg}$ of apixaban taken 6 hours postoperatively twice daily was assessed against $30 \mathrm{mg}$ of enoxaparin administered twice daily, both groups showed extremely low overall VTE rates, but apixaban resulted in significantly less bleeding risk and mortality rate. ${ }^{(72)}$ A meta-analysis, investigated the efficacy of $2.5 \mathrm{mg}$ apixaban or $10 \mathrm{mg}$ rivaroxaban against enoxaparin as prophylaxis after total hip and knee arthroplasty, summarized that oral factor Xa inhibitors were superior to enoxaparin in preventing DVT, but there was no difference in the rate of PE, mortality or postoperative wound infection. ${ }^{(73)}$ Another meta-analysis by Guofeng Ma et al. included 6 RCTs with 13,790 patients showed that the incidence of DVT was significantly decreased with the use of direct Xa inhibitors (both twice daily and once daily regimes) comparing with the enoxaparin treatment $(p<0.01){ }^{(74)}$ However, there was no significant influencing difference between direct Xa inhibitors (twice daily regime) and enoxaparin on the incidence of PE $(p=0.06)$, while significantly lower rate was found for once daily regime of direct Xa inhibitors $(p=0.02)$. With respect to major bleeding, the pooled analysis did not demonstrate a significant difference between groups.

Takeshi FJ et al compared edoxaban, another oral factor $\mathrm{Xa}$ inhibitor, with enoxaparin in thromboprophylaxis following TKA. ${ }^{(75)}$ They concluded that edoxaban $30 \mathrm{mg}$ once daily beginning 6 to 24 hours postoperatively was more effective than subcutaneous enoxaparin $20 \mathrm{mg}$ subcutaneously twice daily beginning 24 to 36 hours postoperatively for 11 to 14 days. This study demonstrated a similar incidence of bleeding events.

Direct thrombin inhibitor (dabigatran) work by binding specifically to the active center of thrombin and inactivate free and fibrin-bound thrombin. This process is reversible leaving small amount of free and active thrombin to control hemostasis. In the US, dabigatran etexilate is a FDA approved oral direct thrombin inhibitor for prevention of atrial fibrillation and stroke, but not for VTE prophylaxis after THA and TKA. Eriksson et al compared dabigatran against enoxaparin (oral dabigatran $220 \mathrm{mg}, 150 \mathrm{mg}$ and subcutaneous enoxaparin $40 \mathrm{mg}$; all taken 6 hours postoperatively then once daily). ${ }^{(76)}$ Efficacy outcomes measured were symptomatic DVT, venographic DVT, and/or symptomatic PE. The safety outcome measured was bleeding events during the course of study. The result showed efficacy outcome of $37.7 \%, 36.4 \%$ and $40.5 \%$ for enoxaparin, $220 \mathrm{mg}$ dabigatran and $150 \mathrm{mg}$ dabigatran, respectively. The major bleeding occurrence did not differ significantly among 3 groups (1.3\% vs $1.5 \%$ vs $1.3 \% ; p>0.05)$.

Warfarin is the first oral anticoagulant that widely used in United States as an anticoagulant agent since 1954. It is vitamin $\mathrm{K}$ antagonist that inhibits the synthesis of active vitamin-K-dependent coagulation factors (factors II, VII, IX and $\mathrm{X}$ as well as protein $\mathrm{C}$ ). Therapeutic anticoagulation is reached 24 to 72 hours after the initial dose. Most commonly, 5 or $10 \mathrm{mg}$ is given the night before or the night of surgery and then dosing is adjusted to maintain an International Normalised Ratio (INR) of about 2.0. Rates of DVT using low-dose warfarin range from $35 \%$ to $59 \%$. ${ }^{(77)}$ INR levels below 2.0 were associated with a four to five times increase in the risk of VTE. ${ }^{(78)}$ When compare warfarin to LMWH, LMWH was a more effective to prevent DVT formation $(p<0.05)$, but no difference to warfarin in preventing symptomatic events including $\mathrm{PE}{ }^{(79,80)}$ The cautions of usage are bleeding risk, potential drug interaction and requirement for constant monitoring. Enoxaparin (low-molecular weight heparin: LMWH) has been widely used for thromboprophylaxis in TJA. Several RCTs reported less DVT and symptomatic PE when compared with placebo. Enoxaparin $40 \mathrm{mg}$ subcutaneously once daily or $30 \mathrm{mg}$ subcutaneously twice daily for 7-14 days postoperatively can reduce the incidence of VTE significantly. ${ }^{(81,82)}$ The bleeding complications trend to increase but not statistically significant. A meta-analysis showed $15 \%-30 \%$ of venographic DVT on discharge date although the TJA patients received enoxaparin for 7-14 days. ${ }^{(83)}$ The recurrent DVT occur 10\%-25\% at 3-4 weeks after THA, while fatal PE can be detected during 1-5 weeks postoperatively. ${ }^{(84)}$ Extended-duration enoxaparin prophylaxis reduced all episodes of DVT and symptomatic venous thromboembolism significantly, especially in THA patients. ${ }^{(87,85)}$ 
Question 17: Does other antiplatelets (not aspirin) have a role for preventing VTE in TJA patient?

Consensus: Other antiplatelets do not have a role for preventing VTE in TJA patient.

Delegate vote: Agree: $70.8 \%$, Disagree: $0 \%$, Abstain: $29.2 \%$ (Strong Consensus)

\section{Justification:}

Currently, other antiplatelets (except aspirin) has not been studied in regard to venous thromboprophylaxis. ${ }^{(86)}$ In theoretically, there are medication with platelet aggregation inhibiting action that little use in the prevention of venous thrombosis, they are essential in the prevention of arterial thrombosis. In TJA patient who continuing other antiplatelets (such as clopidogrel) in the perioperative period can lead to bleeding related complications, so it has been recom- mended that if a patient is to undergo elective surgery and an antiplatelet effect is not desired, should be discontinued at least 5 days prior to surgery.

Question 18: Which conditions should be discontinued chemoprophylaxis of VTE after TJA?

Consensus: If a patient presented with major bleeding or prolonged wound drainage after TJA, chemoprophylaxis of VTE should be discontinued

Delegate vote: Agree: 100\%, Disagree: 0\%, Abstain: 0\% (Unanimous Consensus)

\section{Justification:}

The risk of VTE chemoprophylaxis is associated with bleeding in the perioperative and early post-operative period. There is a potential for long-term problems associated with bleeding, particularly as hematoma is the most important local risk factor for the development of a wound infection after surgery. ${ }^{(87)}$ Saleh et al proposed that both hematoma formation and persistent wound drainage are predicative of superficial surgical site infection, which has a strong correlation with deep-wound infection. ${ }^{(88)}$ Parvizi et al reported the effect of excessive anticoagulation and found that a mean international normalized ratio (INR) of greater than 1.5 was more prevalent in patients who developed post-operative wound complications and subsequent periprosthetic infection and suggested that cautious anticoagulation to prevent periprosthetic infection and its undesirable consequences. ${ }^{(89)}$

Question 19: Is mechanical prophylaxis alone appropriate for VTE prophylaxis in TJA patients who have bleeding disorders?
Consensus: Yes.

Delegate vote: Agree: $91.6 \%$, Disagree: $8.4 \%$, Abstain: $0 \%$ (Strong Consensus)

\section{Justification:}

Although mechanical prophylaxis methods have been shown to reduce the risk of VTE, they have been studied less than the major pharmacological treatment options. Mechanical methods of prophylaxis include graduated compression stocking, intermittent pneumatic compression and venous foot pump. However, $10 \%$ of surgical and medical patients at risk for VTE were classified as being at high risk for bleeding. According to ACCP $9^{\text {th }}$ edition guideline ${ }^{(65)}$, patients who has bleeding disorders or at risk for it and switch them to anticoagulant prophylaxis as soon as bleeding risk is considered to be low and if they met any of the following criteria, which were the strongest predictors of bleeding : active gastroduodenal ulcer, bleeding in the 3 months prior to admission, platelet count $<50000$ or if they had "multiple risk factors" for bleeding of lesser predictive strength, including age $>84$, hepatic failure with INR $>1.5$, severe renal failure, ICU/CCU admission, a central venous catheter in place, rheumatic /autoimmune disease, current cancer and male sex. NICE clinical guideline ${ }^{(90)}$ concluded that the risk factors for bleeding are acquired bleeding disorders (such as acute liver failure), untreated inherited bleeding disorders (such as haemophilia and von Wille brand's disease), thrombocytopenia (platelets less than $75 \times 109 / 1)$, uncontrolled systolic hypertension (230/120 mmh or higher), acute stroke and concurrent use of anticoagulants known (such as warfarin with $\mathrm{INR}>2$ ).

Retrospective study of Morris JK, et al. involved 157 consecutive patients TKA who were treated with bilateral intra- and postoperative intermittent pneumatic compres- sion stockings. ${ }^{(91)}$ All patients were followed for at least 6 weeks postoperatively. Postoperative color duplex ultrasound imaging was obtained for 120 patients 2 to 3 days postoperatively. During hospitalization, $2(1.7 \%)$ patients had acute deep vein thrombosis (DVT) diagnosed, 2 (1.7\%) had DVT of indeterminate age, and 4 (3.3\%) had chronic DVT. During follow-up, $1(0.8 \%)$ patient had an acute DVT diagnosed at 5 weeks postoperatively and $1(0.8 \%)$ had a superficial phlebitis and subsequently had a nonfatal pulmonary embolism 23 days postoperatively. The predominant chemoprophylaxis used was aspirin alone in $107(89.2 \%)$ patients. The results of this study support the use 
of a multimodal approach to VTE prophylaxis in TKA, using bilateral intra and postoperative intermittent pneumatic compression, epidural anesthesia, early mobilization, and postoperative aspirin without the use of major anticoagulation as an effective, safe VTE prophylactic protocol for patients undergoing elective TKA. Retrospective study of Hamilton WG, et al. was to determine the rate of VTE and bleeding complications in a large cohort of patients at a single institution using isolated mechanical thromboembolic prophylaxis in "standard risk" patients, 4,037 TKAs were performed on 3,144 patients at their institution. ${ }^{(92)}$ Mechanical VTE prophylaxis was used for standard risk patients, which included AV impulse foot pumps, thigh high stockings, and early mobilization. Chemoprophylaxis was only given to patients who were at increased thromboembolic risk. The incidence of DVT identified by ultrasound following TKA was 2.1\%. A retrospective review showed 1 patient had a fatal pulmonary embolism, and 5 patients had bleeding complications ( 3 gastrointestinal bleeds, 2 occurred in patients on Coumadin, 1 was in a patient using mechanical prophylaxis only). In addition, there were a severe postoperative wound hematoma in one patient on Coumadin, and an acute knee hemarthrosis six months after surgery in one patient. The result of this study support to use mechanical thromboembolic prophylaxis in risk stratification is safe and effective following TKA and chemoprophylaxis was only given to patients who were at increased thromboembolic risk. Although the supporting evidence to use mechanical prophylaxis alone appropriate for VTE prophylaxis in TJA patients who have bleeding disorders is lacking, avoiding use chemoprophylaxis is good for the safety of patients.

Question 20: Should chemoprophylaxis after knee arthroplasty be given for 2 weeks, if indicated?

Consensus: Yes.

Delegate vote: Agree: $91.6 \%$, Disagree: $8.4 \%$, Abstain: $0 \%$ (Strong Consensus)

\section{Justification:}

The selection of a regimen for venous thromboembolic prophylaxis after total joint arthroplasty is a balance between efficacy and safety. Bleeding may have a negative impact on clinical outcomes. Recently, both the American Academy of Orthopaedic Surgeons (AAOS) and the American College of Chest Physicians (ACCP) developed new evidence-based guidelines for venous thromboembolic prophylaxis after total joint arthroplasty. On the basis of a review of the available literature, the AAOS guideline panel was unable to make a recommendation with respect to the selection of a specific prophylaxis regimen or duration of prophylaxis following routine total joint arthroplasty. The optimal duration of thromboprophylaxis after total knee replacement remains controversial. It is common practice to administer prophylaxis using low-molecular-weight heparin (LMWH) or unfractionated heparin (UFH) until discharge from hospital, usually 7 to 14 days after surgery. ${ }^{(93)}$ International guidelines recommend extending thromboprophylaxis for up to 35 days following major orthopaedic surgery but the recommendation is weak due to moderate quality evidence. In addition, recent oral anticoagulants that exert effect by direct inhibition of thrombin or activated factor $\mathrm{X}$ lack the need for monitoring and have few known drug interactions. ${ }^{(94)}$ Interest in this topic remains high.

Question 21: Shouldchemoprophylaxis after hip arthroplasty be given for 4-5weeks, if indicated?

Consensus: Yes, chemoprophylaxis should be given for 4-5 weeks after hip arthroplasty.

Delegate vote: Agree: 91.6\%, Disagree: $8.4 \%$, Abstain: $0 \%$ (Strong Consensus)

\section{Justification:}

One of the common complications after joint replacement surgery is venous thromboembolism. The risk of developing this complication increases with age, weight and previous history of thromboembolic disease. Incidence of symptomatic venous thromboembolism has been reported in the literature in approximately $2.8 \%$ of patients undergoing hip arthroplasty and $2.1 \%$ of knee arthroplasty patients. The optimal duration of thromboprophylaxis after total hip arthroplasty is still unclear. ${ }^{(95)}$ It is common practice to administer prophylaxis using low-molecularweight heparin (LMWH) or unfractionated heparin (UFH) until discharge from hospital. International guidelines recommend extending thromboprophylaxis for up to 35 days following major orthopaedic surgery. ${ }^{(94)}$

Question 22: Should a thromboprophylaxis be considered in an elderly hip fracture?

Consensus: A thromboprophylaxis should be considered in 
an elderly hip fracture.

Delegate vote: Agree: $100 \%$, Disagree: $0 \%$, Abstain: $0 \%$ (Unanimous Consensus)

\section{Justification:}

Extended (4-week) prophylaxis with fondaparinux can produce a $96 \%$ reduction in risk of DVT and an $89 \%$ reduction in risk of symptomatic VTE events relative to perioperative (1-week) prophylaxis. As the only anticoagulant approved in the United States for thromboprophylaxis in hip fracture patients, fondaparinux offers more effective prophylaxis against VTE without compromising safety. ${ }^{(96)}$

Jeong GK et al, compared the clinical efficacy and side-effect profiles of aspirin, dextran 40, and lowmolecular-weight heparin (enoxaparin) in preventing thromboembolic phenomena after hip fracture surgery. There were 917 patients. Findings included low incidence of thromboembolic phenomena (deep vein thrombosis, $0.5 \%$ $1.7 \%$; pulmonary embolism, $0 \%-2.0 \%$; fatal pulmonary embolism, $0 \%-0.5 \%$ ) and no difference among the 3 pharmacologic agents in thromboembolic prophylaxis efficacy. ${ }^{(97)}$

Question 23: Should we delay the administration of chemoprophylaxis in TJA patient with massive bleeding?

Consensus: The administration of chemoprophylaxis in TJA patient with massive bleeding (defined as blood loss $>500 \mathrm{ml}$ ) should be delayed.

Delegate vote: Agree: 100\%, Disagree: $0 \%$, Abstain: $0 \%$ (Unanimous Consensus)

\section{Justification:}

Following total knee arthroplasty (TKA), only the visible blood loss has been measured. The total blood loss might be underestimated because some hemorrhage is "hidden" or "concealed". Concealed hemorrhage has been an important reason for postoperative blood loss in arthroplasty. It is caused by hemolysis, blood permeating into the interstitial fluid, blood remaining in the joint cavity and the damage of red blood cell caused by the surgical trauma and other factors. ${ }^{(98)}$

Sehat KR et al found that the mean hidden blood loss in THA was about $26 \%$ and visible blood loss was $74 \%$. On the other hand, the mean hidden blood loss in TKA was higher as $51 \%{ }^{(99)}$ If the visible blood loss of patient was more than $500 \mathrm{ml}$ that means the total blood loss would be more than $1,000 \mathrm{ml}$. According to ATLS classification, this amount of hemorrhage has been defined as class II which may increase platelet consumption and result in acute coagulopathy. ${ }^{(100)}$ Acute coagulopathy, characterized by anticoagulation and hyperfibrinolysis, is associated with systemic hypoperfusion. Therefore, the administration of chemoprophylaxis, which is the anticoagulant, in the patients who had massive bleeding (visible blood loss more than $500 \mathrm{ml}$ ) should be delay until hemodynamic stable. ${ }^{(101)}$

Question 24: Does early ambulation decrease the risk of VTE in TJA patients?

Consensus: Yes, early ambulation decreases the risk of VTE in TJA patients.

Delegate vote: Agree: $100 \%$, Disagree: $0 \%$, Abstain: $0 \%$ (Unanimous Consensus)

\section{Justification:}

The ambulation is defined as sitting at bedside, up standing or walking as able. Specific studies have yet to document the value of early ambulation to reduce VTE risk, yet the ICSI work group recommends it for all patients, including those at high risk. ${ }^{(102)}$ Ambulation within 48 hours was associated with a $70 \%$ reduction in the risk of VTE. ${ }^{(103)}$ Several studies demonstrated that early ambulation, a nursing and physical therapy function, directly affects the health care outcome. Although there is no reliable supported evidence, the current AAOS clinical practice guideline recommends that patients should undergo early mobilization following TJA. Early mobilization is of low cost, minimal risk and consistent with current practice. ${ }^{(23)}$

\section{References}

1. Rossi R, Agnelli G. Current role ofvenography in the diagnosis of deep-vein thrombosis. Minerva Cardioangiol 1988; 46: 507-14.

2. Rolfe MW, Solomon DA. Lower extremity venography: still the gold standard. Chest 1999; 116: 853-4.

3. McRae SJ, Ginsberg JS. The diagnostic evaluation of deep vein thrombosis. Am Heart Hosp J 2004; 2: 205-10.

4. American Academy of OrthopedicSurgeons (AAOS) "Deep Vein Thrombosis" [Online]. Available: http://orthoinfo.aaos.org/topic.efm?topic=a00 2192015.

5. Gao FQ, Li ZJ, Zhang K, Huang D, LiuZJ. Risk factors for lower limb swelling after primary total knee arthroplasty. 
Chin Med J (Engl) 201 1; 124: 3896-9.

6. Wells PS, Brill-Edwards P, Stevens P. A novel and rapid whole-blood assay for D-dimer in patients with clinically suspected deep vein thrombosis. Circulation 1995; 91: 2184-87.

7. Freyburger G, Trillaud H, Labrouche S. D-dimer strategy in thrombosis exclusion -- a gold standard study in 100 patients suspected of deep venous thrombosis or pulmonary embolism: 8DD methods compared. Thromb Haemost 1998; 79: 32-7.

8. Brill-Edwards $\mathbf{P}$, Lee $\mathrm{A}$. D-dimer testing in thediagnosis of acute venous thromboembolism. Thromb Haemost 1999; 82: 688-94.

9. Rafee A, Herlikar D, Gilbert R, Stockwell R, McLauchlan G. D-Dimer in the diagnosis of deep vein thrombosis following total hip and knee replacement: A prospective study. Ann R Coll Surg Engl 2008; 90:123-26.

10. Abraham P, Ternisien C, Hubert L, Pidhorz L, Saumet ЛL. Does venous microemboli detection add to the interpretation of D-dimer values following orthopedic surgery? Ultrasound Med Biol 1999; 25: 637-40.

11. Bounameaux H, Miron MJ, Blanchard J, de MP, Hoffmeyer P, Leyvraz PF. Measurement of plasma D-dimer is not useful in the prediction or diagnosis of postoperative deep vein thrombosis in patients undergoing total knee arthroplasty. Blood Coagul Fibrinolysis 1998; 9: 749-52.

12. Niimi R, Hasegawa M, Sudo A, Shi D, YamadaT, Uchida A. Evaluation of soluble fibrin and D-dimer in the diagnosis of postoperative deep vein thrombosis. Biomarkers 2010; 15: 149-57.

13. Bara L, Planes A, Samama MM. Occurrence of thrombosis and haemorrhage, relationship with anti-Xa, anti-IIa activities, and D-dimer plasma levels in patients receiving a low molecular weight heparin, enoxaparin or tinzaparin, to prevent deep vein thrombosis after hip surgery. Br J Haematol 1999; 104: 230-40.

14. Bongard O, Wicky J, Peter R. D-dimer plasma measurement in patients undergoing major hip surgery: use in the prediction and diagnosis of postoperative proximal vein thrombosis. Thromb Res 1994; 74: 487-93.

15. Cofrancesco E, Cortellaro M, Corradi A, RavasiF,
Bertocchi F. Clinical utility of prothrombin fragment $1+2$, thrombin antithrombin III complexes and D-dimer measurements in the diagnosis of deep vein thrombosis following total hip replacement. Thromb Haemost 1998; 79: 509-10.

16. Maezawa K, Nozawa M, Aritomi K, Kubota M, Shitoto $\mathrm{K}$, Kurosawa H. Changes of D- dimer after total hip arthroplasty in patients with and without intraoperative heparin. Arch Orthop Trauma Surg 2008; 128: 37-40.

17. Shiota N, Sato T, Nishida K. Changes in LPIAD-dimer levels after total hip or knee arthroplasty relevant to deep-vein thrombosis diagnosed by bilateral ascending venography. J Orthop Sci 2002; 7: 444-50.

18. Yoo MC, Cho YJ, Ghanem E, Ramteke A, Kim KI. Deep vein thrombosis after total hip arthroplasty in Korean patients and D-dimer as a screening tool. Arch Orthop Trauma Surg 2009; 129: 887-94.

19. Lensing AW, Prandoni P, Brandjes D. Detectionof deep-vein thrombosis by real-time B-mode ultrasonography. N Engl J Med 1989; 320: 342-45.

20. Mattos MA, Londrey GL, Leutz DW. Color-flow duplex scanning for the surveillance and diagnosis of acute deep venous thrombosis. J VascSurg 1992; 15: 366-75.

21. Le Gal G, Righini M, Sanchez O. A positive compression ultrasonography of the lower limb veins is highly predictive of pulmonary embolism on computed tomography in suspected patients. Thromb Haemost 2006; 95: 963-66.

22. Schellong SM, Beyer J, Kakkar AK. Ultrasound screening for asymptomatic deep vein thrombosis after major orthopaedic surgery: the VENUS study. J Thromb Haemost 2007; 5: 1431-7.

23. Mont MA, Jacobs JJ, Boggio LN, Bozic KJ, DellaValle $\mathrm{CJ}$, Goodman SB, et al. AAOS. Preventing venous thromboembolic disease in patients undergoing elective hip and knee arthroplasty, evidence-based guideline? and evidence report. J Am Acad Orthop Surg 2011; 19: 768-76.

24. Schmidt B, Michler R, Klein M, Faulmann G, Weber C, Schellong S. Ultrasound screening for distal vein thrombosis is not beneficial after major orthopedic surgery. A randomized controlled trial. Thromb Haemost 2003; 90: 949-54. 
25. Pellegrini VD Jr, Donaldson CT, Farber DC. TheMark Coventry Award: Prevention of readmission for venous thromboembolism after total knee arthroplasty. Clin Orthop Relat Res 2006; 452: 21-7.

26. Jacobs JJ, Mont MA, Bozic KJ, Della Valle CJ, Goodman SB, Lewis CG, et al. American Academy of Orthopaedic Surgeons clinical practice guideline on: preventing venous thromboembolic disease in patients undergoing elective hip and knee arthroplasty. J Bone Joint Surg Am 2012; 94: 746-7.

27. Nejat EJ, Polotsky AJ, Pal L. Predictors of chronic disease at midlife and beyond the health risks of obesity. Maturitas 2010; 65: 106-11.

28. Kabrhel C, Varraso R, Goldhaber SZ, Rimm EB, Camargo CA. Prospective study of BMI and the risk of pulmonary embolism in women. Obesity 2009; 17 : 2040-46.

29. Eichinger S, Hron G, Bialonczyk C, Hirschl M, Minar E, Wagner $\mathrm{O}$, et al. Overweight, obesity, and the risk of recurrent venous thromboembolism. Arch Int Med 2008; 168: 1678-83.

30. Severinsen MT, Kristensen SR, Johnsen SP, Dethlefsen C, Tjonneland A, Overvad K. Anthropometry, body fat, and venous thromboembolism: A Danish follow-up study. Circulation 2009; 120: 1850-57.

31. Barba R, Zapatero A, Losa JE, Valdes V, TodoliJA, Di Micco P, et al. Body mass index and mortality in patients with acute venous thromboembolism: Findings from the RIETE registry. J Thromb Haemost 2008; 6: 595-600.

32. Stein PD, Matta F, Goldman J. Obesity andpulmonary embolism: The mounting evidence of risk and the mortality paradox. Thromb Res 2011; 128: 518-23.

33. Kalantar-Zadeh K, Horwich TB, Oreopoulos A, Kovesdy CP, Younessi H, Anker SD, et al. Risk factor paradox in wasting diseases. Curr Opin Clin Nutr Metab Care 2007; 10: 433-42.

34. Garcia-Raso A, Llamas Sillero P. Elevated body fat is a risk factor for venous thromboembolism and thrombotic complications. Epidemiol Rep 2014; 2: 3.

35. Eikelboom JW, Weitz JI. Importance of family history as a risk factor for venous thromboembolism. Circulation 2011; 124: 996-97.

36. Osinbowale O, Ali L, Chi YW. Venous thromboem bolism: A clinical review. Postgrad Med 2010; 122: 54-65.
37. Martin GM, Thornhill TS, Katz JN. Complications of total knee arthroplasty. In: Post TW, editor. UpToDate. Waltham, MA: UpToDate; 2015.

38. Erens GA, Thornhill TS, Katz JN. Complicationsafter total hip arthroplasty. In: Post TW, editor. UpToDate. Waltham, MA: UpToDate; 2015.

39. Kanchanabat B, Stapanavatr W, Meknavin S, Soorapanth C, Sumanasrethakul C, Kanchanasuttirak P. Systematic review and meta-analysis on the rate of postoperative venous thromboembolism in orthopaedic surgery in Asian patients without thromboprophylaxis. Br J Surg 2011;98: 1356-64.

40. ChotanaphutiT, Ongnamthip P, Silpipat S, Foojareonyos $\mathrm{T}$, Roschan S, Reumthantong A. The prevalence of thrombophilia and venous thromboembolism in total knee arthroplasty. J Med Assoc Thai 2007; 90: 1342-7.

41. CharoencholvanichK, Siriwattanasakul P, Tranexamic acid reduces blood loss and blood transfusion after TKA: A prospective randomized controlled trial. Clin Orthop Relat Res 2011; 469: 2874-80.

42. Gandhi R. Tranexamic acid and the reduction of blood loss in total knee and hip arthroplasty: a meta-analysis. BMC Res Notes 2013; 6: 184.

43. Lin ZX, Woolf SK. Safety, efficacy, and costeffectiveness of tranexamic acid in orthopedic surgery. Orthopedics 2016; 39:119-30.

44. Haake DA, Berkman SA. Venous thromboembolic disease after hip surgery. Risk factors, prophylaxis and diagnosis. Clin Orthop 1989; 242: 212-31.

45. Todd CJ, Freeman CJ, Camilleri-Ferrante C, Palmer CR, Hyder A, Laxton CE, et al. Differences in mortality after fracture of the hip: the East Anglian audit. Br Med J 1995; 310: 904-8.

46. Zahn HR, Skinner JA, Porteous MJ. The preoperative prevalence of deep vein thrombosis in patients with femoral neck fractures and delayed operation. Injury 1999; 30: 605-7.

47. Hefley FG Jr, Nelson CL, Puskarich-May CL. Effect of delayed admission to the hospital on the preoperative prevalence of deep-vein thrombosis associated with fractures about the hip. J Bone Joint Surg Am 1996; 78: 581-3.

48. Lee S, Hwang J, Kim Y, Yoo JJ. Venous thromboembolism following hip and knee replacement 
arthroplasty in Korea: A nationwide study based on claims registry. J Korean Med Sci 2016; 31: 80-8.

49. Hu S, Zhang ZY, Hua YQ, Li J, Cai ZD. A comparison of regional and general anaesthesia for total replacement of the hip or knee: a meta-analysis. J Bone Joint Surg Br 2009; 91: 935-42.

50. Atchabahian A, Schwartz G, Hall CB, Lajam CM, Andreae $\mathrm{MH}$. Regional analgesia

for improvement of long-term functional outcome after elective large joint replacement. Cochrane Database Syst Rev 2015; 8: CD010278.

51. Caprini JA, Arcelus J, Reyna JJ. Effective risk stratification of surgical and nonsurgical patients for venous thromboembolic disease. Semin Hematol 2001; 38 (2Suppl5): 12-9.

52. Haas SB, Barrack RL, Westrich G. Venous thromboembolic disease after total hip and knee arthroplasty. Instr Course Lect 2009; 58: 781-93.

53. Westrich GH, Haas SB, Mosca P, Peterson $M$. Meta-analysis of thromboembolic prophylaxis after total knee arthroplasty. J Bone Joint Surg Br 2000; 82: 795-800.

54. Hernandez AJ, Almeida AM. The influence of tourniquet use and operative time on the incidence of deep vein thrombosis in total knee arthroplasty. Clin Sei 2012; 67: 1053-57.

55. Lacut K. Mechanical prophylaxis of venous thromboembolism Rev Prat 2011; 61: 1232-36.

56. Leme LEG, Sguizzatto GT. Prophylaxis of venous thromboembolism in orthopaedic surgery. Rev Bras Ortop 2012; 47: 685-93.

57. MacLellan DG, Fletcher JP. Mechanical compression in the prophylaxis of venous thromboembolism. ANZ J Surg 2007; 77: 418-23.

58. Parvizi J, Azzam K, Rothman RH. Deep venous thrombosis prophylaxis for total joint arthroplasty: American Academy of Orthopaedic Surgeons guidelines. J Arthroplasty 2008; 23(7Suppl): 2.

59. Sharrock NE, Gonzalez Della Valle A, Go G, Lyman S, Salvati EA. Potent anticoagulants are associated with a higher all-cause mortality rate after hip and knee arthroplasty. Clin Orthop Relat Res 2008; 466: 714.

60. Walsh M, Preston C, Bong M, Patel V, Di Cesare PE. Relative risk factors for requirement of blood transfusion after total hip arthroplasty. J Arthroplasty 2007; 22: 1162.

61. Freedman KB, Brookenthal KR, Fitzgerald JrRH, Williams S, Lonner JH. A meta-analysis of thromboembolic prophylaxis following elective total hip arthroplasty. J Bone Joint Surg Am 2000; 82-A: 929.

62. Kim YH, Kulkarni SS, Park JW, Kim JS. Prevalence of deep vein thrombosis and pulmonary embolism treated with mechanical compression device after total knee arthroplasty in Asian patients. J Arthroplasty 2015; 30: 1633-37.

63. Lee WS, Kim KI, Lee HJ. The incidence of pulmonary embolism and deep vein thrombosis after knee arthroplasty in Asians remain low. A meta-analysis. Clin Orthop Relat Res 2013; 471: 1523.

64. Pour AE, Keshavarzi NR, Purtill JJ, Sharkey PF,Parvizi $\mathbf{J}$. Is venous foot pump effective in prevention of thromboembolic disease after joint arthroplasty: a metaanalysis. J Arthroplasty 2013; 28: 410-7.

65. Falck-Ytter Y, Francis CW, Johanson NA, Curley C, Dahl OE, Schulman S, et al. Prevention of VTE in orthopedic surgery patients: antithrombotic therapy and prevention of thrombosis: American College of Chest Physicians evidence-based clinical practice guidelines. Chest $2012 ; 141$ (2suppl): e278S-325S.

66. An VV, Phan K, Levy YD, Bruce WJ. Aspirin as thromboprophylaxis in hip and knee arthroplasty: a systematic review and meta-analysis. J Arthroplasty 2016; 31:2608-16.

67. Rodgers A, MacMahon S, Collins R, Prentice C. Prevention of pulmonary embolism and deep vein thrombosis with low dose aspirin: Pulmonary embolism prevention (PEP) trial. Lancet 2000; 355: 1295-302.

68. Drescher FS, Sirovich BE, Lee A, Morrison DH, Chiang WH, Larson RJ. Aspirin versus anticoagulation for prevention of venous thromboembolism major lower extremity orthopedic surgery: a systematic review and meta-analysis. J Hosp Med 2014; 9:579-85.

69. Schousboe JT, Brown GA. Cost-effectiveness of low-molecular-weight heparin compared with aspirin for prophylaxis against venous thromboembolism after total joint arthroplasty. J Bone Joint Surg Am 2013; 95-A: 1256-64.

70. Lassen MR, Ageno W, Borris LC, Lieberman JR, Rosencher N, Bandel TJ, et al. Rivaroxaban vs enoxaparin 
for thromboprophylaxis after total knee arthroplasty. $\mathrm{N}$ Engl J Med 2008; 358: 2776-86.

71. Eriksson BI, Borris LC, Friedman RJ,Haas S, HuismanMV, Kakkar AK, et al. Rivaroxaban vs enoxaparin for thromboprophylaxis after hip arthroplasty. N Engl J Med 2008; 358: 2765-75.

72. Lassen MR, Raskob GE, Gallus A, PineoG, Chen D, Portman RJ. Apixaban or enoxaparin for thromboprophylaxis after knee replacement. N Engl J Med 2009; 361: 594-604.

73. Russell RD, Huo MH. Apixaban and rivaroxaban decrease deep vein thrombosis but not other complications after total hip and total knee arthroplasty. J Arthroplasty 2013;28: 1477-81.

74. Ma G, Zhang R, Wu X, Wang D, Ying K.Direct factor $\mathrm{Xa}$ inhibitors (rivaroxaban and apixaban) versus enoxaparin for the prevention of venous thromboembolism after total knee replacement: A meta-analysis of 6 randomized clinical trials. Thromb Res 2015; 135 : 816-22.

75. Takeshi FJ, Ching-Jen W, Yohko K, MashioN, Tetsuya $\mathrm{K}$, Kei I, et al. Safety and efficacy of edoxaban, an oral factor xa inhibitor, versus enoxaparin for thromboprophylaxis after total knee arthroplasty: the STARS E-3 trial: A randomized, double-blind, double-dummy study. Thromb Res 2014; 134: 1198-204.

76. Eriksson BI, Dahl OE, Rosencher N, Kurth AA, Van Dijk CN, Frostick SP. Oral dabigatranetexilate vs subcutaneous enoxaparin for the prevention of venous thromboembolism after total knee replacement: the RE-MODEL randomized trial. J Thromb Haemost 2007; 5: 2178-85.

77. Lotke PA, Palevsky H, Keenan AM. Aspirin and warfarin for thromboembolic disease after total joint arthroplasty. Clin Orthop 1996; 324: 251-8.

78. Nordstrom BL, Kachroo S, Fraeman KH, Nutescu EA, Schein JR, Fisher A, et al. Warfarin prophylaxis in patients after total knee or hip arthroplasty -international normalized ratio patterns and venous thromboembolism. Curr Med Res Opin 2011; 27:10: 1973-85.

79. Heit JA, Berkowitz SD, Bona R, Cabanas V, Corson JD, Elliott CG. Efficacy and safety of low molecular weight heparin (ardeparin sodium) compared with warfarin for the prevention of venous thromboembolism after total knee replacement surgery: a double-blind, dose-ranging study. Thromb Haemost 1997; 77: 32-8.

80. Fitzgerald Jr RH, Spiro TE, Trowbridge AA, Gardiner Jr GA, Whitsett TL, O'Connell MB, et al. Prevention of venous thrombo-embolic disease following primary total knee arthroplasty. A randomized, multicenter, openlabel, parallel-group comparison of enoxaparin and warfarin. J Bone Joint Surg Am 2001; 83: 900-6.

81. Leclerc JR, Geerts WH, Desjardins L. Prevention of deep vein thrombosis after major knee surgery-a randomized, double-blind trial comparing a low molecular weight heparin fragment (enoxaparin) to placebo. Thromb Haemost 1992; 67: 417-23.

82. Turpie AG, Levine MN, Hirsh J. Arandomized controlled trial of a low-molecular-weight heparin (enoxaparin) to prevent deep-vein thrombosis in patients undergoing elective hip surgery. N Engl J Med 1986; 315: 925-9.

83. Eikelboom JW, Quinlan DJ, Douketis JD. Extended duration prophylaxis against venous thromboembolism after total hip or knee replacement: a meta-analysis of the randomised trials. Lancet 2001; 358: 9-15.

84. Planes A, Vochelle N, Darmon JY, Fagola M, Bellaud M, Huet Y. Risk of deep-venous thrombosis after hospital discharge in patients having undergone total hip replacement: double-blind randomized comparison of enoxaparin versus placebo. Lancet 1996; 348: 224-28.

85. Hull RD, Pineo GF, Stein PD. Extended out-of-hospital low-molecular-weight heparin prophylaxis against deep venous thrombosis in patients after elective hip arthroplasty: a systematic review. Ann Intern Med 2001; 135: 858-69.

86. Steele MJ, Fox JS, Fletcher JP, Grigg LE, Bell G. Clopidogrel dilemma for orthopaedic surgeons. ANZ J Surg 2011; 81: 774-84.

87. Anielski R, Barczynski M. [Postoperative wound infections. II. Risk factors related to surgery]. Przegl Lek 1998; 55: 109-19.

88. Saleh K, Olson M, Resig S. Predictors of wound infection in hip and knee joint replacement: results from a 20-year surveillance program. J Orthop Res 2002; 20: 506-15.

89. Parvizi J, Ghanem E, Joshi A, Sharkey PF,Hozack WJ, Rothman RH. Does 'excessive' anticoagulation predispose 
to periprosthetic infection? J Arthroplasty 2007; 22 (Suppl2) : 24-8.

90. Venous thromboembolism; Reducing the risk for patients in hospital. NICE Clinical guideline 2012 (National Institute for Health and Clinical Excellence).

91. Morris JK, Fincham BM. Intermittent pneumatic compression for venous thromboembolism prophylaxis in total knee arthroplasty. Orthopedics 2012; 35:1716-21.

92. Hamilton WG, Reeves JD, Fricka KB, Goyal N, Engh GA, Parks NL. Mechanical thromboembolic prophylaxis with risk stratification in total knee arthroplasty. J Arthroplasty 2015; 30: 43-5.

93. Lieberman JR, Pensak MJ. Prevention of venous thromboembolic disease after total hip and knee arthroplasty. J Bone Joint Surg Am 2013; 95: 1801-11.

94. Forster R, Stewart M. Anticoagulants (extended duration) for prevention of venous thromboembolism following total hip or knee replacement or hip fracture repair. Cochrane Database Syst Rev 2016; 3.CD004179.

95. Farfan M, Bautista M, Bonilla G, Rojas J, Llinás A, Navas J. Worldwide adherence to ACCP guidelines for thromboprophylaxis after major orthopedic surgery: A systematic review of literature and meta-analysis. Thromb Res 2016; 141: 163-70.

96. Kwong LM. Hip fracture and venous thromboembolism in the elderly. J Surg Orthop Adv 2004; 13: 139-48.
97. Jeong GK, Gruson KI, Egol KA, Aharonoff GB, Karp AH, Zuckerman JD, Koval KJ. Thromboprophylaxis after hip fracture: evaluation of 3 pharmacologic agents. Am J Orthop (Belle Mead NJ) 2007; 36: 135-40.

98. Qi Z, Yiqin Z, Haishan W, Yuli W, Qirong Q, Hui Z, et al. Changes of hemoglobin and hematocrit in elderly patients receiving lower joint arthroplasty without allogeneic blood. Chi Medical J 2015; 128: 75-8.

99. Sehat KR, Evans RL, Newman JH. Hidden blood loss following hip and knee arthroplasty. J Bone Joint Surg Br 2004; 86-B: 561-5.

100. Stainsby D, MacLennan S, Thomas D, Isaac J, Hamilton PJ. Guidelines on the management of massive blood loss. Br J Haematol 2006; 135: 634-41.

101. Brohi K, Cohen MJ, Ganter MT, Schultz MJ, Levi M, Mackersie RC, et al. Acute coagulopathy of trauma: Hypoperfusion induces systemic anticoagulation and hyperfibrinolysis. J Trauma 2008; 64: 1211-7.

102. National Guideline Clearing house (NGC) and the Institute for Clinical Systems Improvement (ICSI). Venous thromboembolism prophylaxis. Nov 2003

103. Szekendi M. Early ambulationr the risk of venous thromboembolism after total knee replacement. ANA $7^{\text {th }}$ Annual Nursing Quality. Conference, February 2013. 